TRANSACTIONS OF THE

AMERICAN MATHEMATICAL SOCIETY

Volume 362, Number 8, August 2010, Pages 4267-4282

S 0002-9947(10)04947-0

Article electronically published on March 1, 2010

\title{
LYAPUNOV EXPONENTS OF HYPERBOLIC MEASURES AND HYPERBOLIC PERIODIC ORBITS
}

\author{
ZHENQI WANG AND WENXIANG SUN
}

\begin{abstract}
Lyapunov exponents of a hyperbolic ergodic measure are approximated by Lyapunov exponents of hyperbolic atomic measures on periodic orbits.
\end{abstract}

\section{INTRODUCTION}

Let $f$ be a $C^{1+\alpha}, \alpha>0$, diffeomorphism of a compact $d$-dimensional manifold $M$ and $D f: T M \rightarrow T M$ the derivative of $f$. Let us fix a smooth Riemannian metric on $M$, i.e., a scalar product (and consequently a norm) in every tangent space $T_{x} M, x \in M$ which depends on $x$ in a differentiable way. The limit

$$
\lambda(x, v)=\lim _{n \rightarrow \infty} \frac{\log \left\|D f^{n} v\right\|}{n}, v \in T_{x} M, v \neq 0, x \in M
$$

is called a Lyapunov exponent for a tangent vector $v \in T_{x} M$. Lyapunov exponents describe the asymptotic evolution of a tangent map: positive or negative exponents correspond to exponential growth or decay of the norm, respectively, whereas vanishing exponents mean lack of exponential behavior. From the Oseledec theorem [8], the limit $\lambda(x, v)$ exists for all nonvanishing vectors $v$ based on almost all state points $x$ in $M$ with respect to any given invariant measure, and it is independent of the points if the measure is ergodic. The function $\lambda$ being defined on the tangent bundle $T M$ takes on at most $d$ values on each tangent space $T_{x} M$. None of these values depends on the choice of a Riemannian metric. Back to Lyapunov and Perron, Lyapunov exponents for a differential equation are a natural generalization of the eigenvalues of the matrix in the linear part of the equation, and the condition that all Lyapunov exponents are negative together with the Lyapunov-Perron regularity implies Lyapunov stability; see Chapter 1 in 1 for the definition of Lyapunov-Perron regularity and the classical theory of Lyapunov stability. The abstract Lyapunov exponent defined in (1.1) is a basic concept and an active topic in the theory of nonuniformly hyperbolic systems known as Pesin theory. Pesin theory recovers some hyperbolic behavior for the points whose Lyapunov exponents are all nonzero. In particular, these points have well-defined unstable and stable invariant

Received by the editors June 11, 2008.

2000 Mathematics Subject Classification. Primary 37C40, 37D25.

Key words and phrases. Lypunov exponent, Pesin set, hyperbolic measure.

The second author was supported by NNSFC (\# 10231020, 10671006) and the National Basic Research Program of China(973 Program) (\# 2006CB805900). The second author was the corresponding author for this paper. 
manifolds. For these reasons, an ergodic invariant measure is called hyperbolic if its Lyapunov exponents are different from zero.

The closing lemma of Katok [5] in Pesin theory states that hyperbolic periodic points are dense in the closure of the basin of a given hyperbolic measure. Based on this lemma we will show in the present paper that the Lyapunov exponents of a hyperbolic ergodic measure are approximated by those of a hyperbolic atomic measure on a periodic orbit. Lyapunov exponents for an atomic measure concentrated on a periodic orbit with period $p$ are exactly the logarithm of the norms of eigenvalues for $D f^{p}$. Now we state our main theorem in the present paper.

Theorem 1.1. Let $f$ be a $C^{1+\alpha}$ diffeomorphism of a compact d-dimensional Riemannian manifold $M$ preserving an ergodic hyperbolic measure $m$ with Lyapunov exponents $\lambda_{1} \leq \ldots \leq \lambda_{r}<0<\lambda_{r+1} \leq \ldots \leq \lambda_{d}$. Then the Lyapunov exponents of $m$ can be approximated by the Lyapunov exponents of hyperbolic periodic orbits. More precisely, for any $\gamma>0$, there exists a hyperbolic periodic point $z$ with Lyapunov exponents $\lambda_{1}^{z} \leq \ldots \leq \lambda_{d}^{z}$ such that $\left|\lambda_{i}-\lambda_{i}^{z}\right|<\gamma, i=1, \ldots, d$.

It is known from S. Theorem 5.5 in [6] (see also Theorem 15.4.7 in [2]) that the smallest absolute value in all of the Lyapunov exponents of a hyperbolic measure can be approximated from the upper side by the smallest absolute value in all of the Lyapunov exponents of a hyperbolic periodic orbit. Our Theorem 1.1 could be viewed as a generalization of this result. Moreover, our result may contribute to a strong version of the closing lemma in [7, which states that a recurrent orbit in a Pesin set of a hyperbolic measure and the Oseledec splitting it carries can be approximated by hyperbolic periodic orbits and their Oseledec splittings. Due to the discontinuity of the Oseledec splitting, this is a nontrivial topic.

A classical result of Sigmund [14 in uniform hyperbolic systems states that periodic measures are dense in the set of invariant measures. For the nonuniform hyperbolic case, Hirayama 3 proved that periodic measures are dense in the set of invariant measures supported by a total measure set with respect to a hyperbolic mixing measure. Quite recently, Liang, Liu and Sun [7] replaced the assumption of a hyperbolic mixing measure by a more natural and weaker assumption of a hyperbolic ergodic measure and generalized Hirayama's result. It is good to point out that this series of results of measure approximation does not imply automatically the present work of exponent approximation.

Using preliminary facts recalled in Section 2, we prove in Section 3 that the largest (smallest) Lyapuov exponent of an ergodic hyperbolic measure $m$ can be approximated by that of atomic measures on hyperbolic periodic orbits. A similar approximation property for the 2nd-exterior power shows that the sum of the largest (smallest) two Lyapunov exponents of $m$ can be approximated by that of hyperbolic periodic orbits, which then implies that the two largest (smallest) Lyapunov exponents of $m$ can be approximated by those of hyperbolic periodic orbits. Inductive arguments show Theorem 1.1] in Section 4.

\section{Preliminaries}

In this section, we recall preliminary facts cited from reference papers and books.

2.1. A criterion for hyperbolicity (4]). We denote by $M$ a $C^{\infty}$ compact manifold throughout this paper. Let $U \subseteq M$ be an open set and let $f$ be a $C^{1}$ diffeomorphism from $U$ onto $f(U)$. Let $\triangle \subset U$ be a compact and $f$-invariant set. Let 
$T_{\Delta} M=E^{1} \oplus E^{2}$ be a Whitney splitting. Put

$$
\begin{gathered}
D f=\left[\begin{array}{ll}
G_{11} & G_{12} \\
G_{21} & G_{22}
\end{array}\right]: E^{1} \oplus E^{2} \rightarrow E^{1} \oplus E^{2}, \\
D f^{-1}=\left[\begin{array}{ll}
G_{11}^{\prime} & G_{12}^{\prime} \\
G_{21}^{\prime} & G_{22}^{\prime}
\end{array}\right]: E^{1} \oplus E^{2} \rightarrow E^{1} \oplus E^{2},
\end{gathered}
$$

where $G_{i j}, G_{i j}^{\prime}, i, j=1,2$ are bundle maps covering $f$. If there exist $0<\lambda<1$ and $\epsilon>0$ satisfying $0<\varepsilon<\min \left\{1-\lambda, \lambda^{-1}-1\right\}$, and also

$$
\begin{gathered}
\max \left\{\left|G_{11}\right|,\left|G_{22}^{-1}\right|,\left|G_{11}^{\prime-1}\right|,\left|G_{22}^{\prime}\right|\right\}<\lambda, \\
\max \left\{\left|G_{12}\right|,\left|G_{21}\right|,\left|G_{12}^{\prime}\right|,\left|G_{21}^{\prime}\right|\right\}<\varepsilon,
\end{gathered}
$$

then $f: \Delta \rightarrow \Delta$ is hyperbolic.

2.2. Oseledec theorem ([8]). Let $d$ denote the dimension of the compact manifold $M$. Let $f: M \rightarrow M$ be a $C^{1}$ diffeomorphism preserving an ergodic probability measure $m$. Then there exist

(a) real numbers $\lambda_{1}<\cdots<\lambda_{k}(k \leq d)$;

(b) positive integers $n_{1}, \ldots, n_{k}$, satisfying $n_{1}+\cdots+n_{k}=d$;

(c) a Borel set $L(m)$ satisfying $f L(m)=L(m)$ and $m(L(m))=1$;

(d) a measurable splitting $T_{x} M=E_{x}^{1} \oplus \cdots \oplus E_{x}^{k}$ with $\operatorname{dim} E_{x}^{i}=n_{i}$ and $D f\left(E_{x}^{i}\right)=$ $E_{f x}^{i}$, such that

$$
\lim _{n \rightarrow \pm \infty} \frac{\log \left\|D f^{n} v\right\|}{n}=\lambda_{i}
$$

for $\forall x \in L(m), v \in E_{x}^{i}, i=1,2, \ldots, k$.

The set $L(m)$ is called an Oseledec basin of $m$.

2.3. Parallelepiped spectrum (see [13]). Let $f: M \rightarrow M$ be a $C^{1}$ diffeomorphism preserving an ergodic measure $m$. The Lyapunov exponents of $m$ being $\lambda_{1}<\ldots<\lambda_{k}$ with associated splitting $T_{x} M=E^{1} \oplus \cdots \oplus E^{k}, x \in L(m)$ and multiplicities $\Gamma(r)=\operatorname{dim} E^{r}$ constitute the spectrum of $(m, D f)$. We construct a bundle $\Lambda^{i}(M), 2 \leq i \leq d$ (recall $\left.d=\operatorname{dim} M\right)$ of $C_{d^{-}}^{i}$ dimension on $M$, where the fiber over $x$ is

$$
\Lambda^{i}(x)=\left\{v_{j_{1}} \wedge \ldots \wedge v_{j_{i}}: v_{j_{k}} \in T_{x} M, 1 \leq k \leq i, 1 \leq j_{1}<j_{2}<\ldots<j_{i} \leq d\right\} .
$$

Let $D f^{\Lambda^{i}}: \Lambda^{i}(M) \rightarrow \Lambda^{i}(M)$ denote the $i$-exterior power of $D f$, namely,

$$
D_{x} f^{\Lambda^{i}}\left(v_{j_{1}} \wedge \ldots \wedge v_{j_{i}}\right)=D_{x} f\left(v_{j_{1}}\right) \wedge \ldots \wedge D_{x} f\left(v_{j_{i}}\right) .
$$

We define a norm \|\|$_{\Lambda^{i}}$ on $\Lambda^{i}$ by assigning $v_{j_{1}} \wedge \ldots \wedge v_{j_{i}}$ to the $i$-volume of the parallelepiped generated by the vectors $v_{j_{1}}, \ldots, v_{j_{i}}$. The spectrum of Lyapunov exponents of $\left(m, D f^{\Lambda^{i}}\right)$ consists of numbers $\varpi=\sum_{r} n_{r} \lambda_{r}$, where $0 \leq n_{r} \leq \Gamma(r)$ and $\sum_{r} n_{r}=i$. The subspace corresponding to $\varpi$ in the associated splitting is generated by $v_{j_{1}} \wedge \ldots \wedge v_{j_{i}}$, where $v_{j_{l}} \in E^{j_{l}}$ and $\sum_{l=1}^{i} \lambda^{j_{l}}=\varpi$. 
2.4. Pesin set (9-11]). Given $\lambda, \mu \gg \varepsilon>0$, and for all $k \in \mathbb{Z}^{+}$, we define $\Lambda_{k}=\Lambda_{k}(\lambda, \mu ; \varepsilon)$ to be all points $x \in M$ for which there is a splitting $T_{x} M=E_{x}^{s} \oplus E_{x}^{u}$ with invariant property $\left(D_{x} f^{m}\right) E_{x}^{s}=E_{f^{m} x}^{s}$ and $\left(D_{x} f^{m}\right) E_{x}^{u}=E_{f^{m} x}^{u}$ and satisfying:

(a) $\left\|D f^{n} / E_{f}^{s} m_{x}\right\| \leq e^{\varepsilon k} e^{-(\lambda-\varepsilon) n} e^{\varepsilon|m|}, \forall m \in \mathbb{Z}, n \geq 1$;

(b) $\left\|D f^{-n} / E_{f m_{x}}^{u}\right\| \leq e^{\varepsilon k} e^{-(\mu-\varepsilon) n} e^{\varepsilon|m|}, \forall m \in \mathbb{Z}, n \geq 1$;

(c) $\tan \left(\operatorname{Angle}\left(E_{f^{m} x}^{s}, E_{f^{m} x}^{u}\right)\right) \geq e^{-\varepsilon k} e^{-\varepsilon|m|}, \forall m \in \mathbb{Z}$.

We put $\Lambda=\Lambda(\lambda, \mu ; \varepsilon)=\bigcup_{k=1}^{+\infty} \Lambda_{k}$ and call $\Lambda$ a Pesin set.

Let $m$ be an ergodic hyperbolic measure preserved by $f$. We denote by $\lambda$ the absolute value of the largest negative Lyapunov exponent and by $\mu$ the smallest positive Lyapunov exponent of $m$. Let $E^{s}$ and $E^{u}$ denote, respectively, the direct sum of the subbundles corresponding to negative Lyapunov exponents and the sum of subbundles corresponding to positive exponents. Then $E^{s}$ and $E^{u}$ are well defined on the Oseledec basin $L(m)$ (see $\S 2.2$ ), they are $D f$ invariant and their direct sum based on $L(m)$ coincides with $T_{L(m)} M$. By using these $\lambda$ and $\mu$ together with $E^{s}$ and $E^{u}$ we get as in the above definition a Pesin set $\Lambda=\Lambda(\lambda, \mu ; \varepsilon)$ for small $\varepsilon$. This is called a Pesin set of $m$. It follows that $m(\Lambda \backslash L(m))+m(L(m) \backslash \Lambda)=0$.

The following statements are elementary:

(1) $\Lambda_{1} \subseteq \Lambda_{2} \subseteq \Lambda_{3} \subseteq \cdots$;

(2) $f\left(\Lambda_{k}\right) \subseteq \Lambda_{k+1}, f^{-1}\left(\Lambda_{k}\right) \subseteq \Lambda_{k+1}$;

(3) $\Lambda_{k}$ is compact for $\forall k \geq 1$;

(4) for $\forall k \geq 1$ the splitting $x \rightarrow E_{x}^{u} \oplus E_{x}^{s}$ depends continuously on $\Lambda_{k}$.

2.5. Lyapunov metric \|\|$\left.\left.^{\prime}(9]-11\right]\right)$. Let $\lambda^{\prime}=\lambda-2 \varepsilon, \mu^{\prime}=\mu-2 \varepsilon$. Note that $\epsilon \ll \lambda, \mu$. Then $\lambda^{\prime}, \mu^{\prime}>0$. Let $x \in \Lambda(\lambda, \mu, \epsilon)$, a Pesin set; see $\S 2.4$. For $v_{s} \in$ $E_{x}^{s}$, we define $\left\|v_{s}\right\|_{s}=\sum_{n=0}^{+\infty} e^{\lambda^{\prime} n}\left\|D_{x} f^{n}\left(v_{s}\right)\right\|$; for $v_{u} \in E_{x}^{u}$, we define $\left\|v_{u}\right\|_{u}=$ $\sum_{n=0}^{+\infty} e^{\mu^{\prime} n}\left\|D_{x} f^{-n}\left(v_{u}\right)\right\|$, and we define the Lyapunov metric \|\|$^{\prime}$ on $T_{\Lambda} M$ by $\|v\|^{\prime}=$ $\max \left\{\left\|v_{s}\right\|_{s},\left\|v_{u}\right\|_{u}\right\}$, where $v=v_{s}+v_{u} \in E_{x}^{s} \oplus E_{x}^{u}, x \in \Lambda$. As usual we call the norm $\|.\|^{\prime}$ a Lyapunov metric. This metric is in general not equivalent to the Riemannian metric. With the Lyapunov metric, $f: \Lambda \rightarrow \Lambda$ is uniformly hyperbolic. The following estimates are known:

(a) $\left\|D f / E_{x}^{s}\right\|^{\prime} \leq e^{-\lambda^{\prime}},\left\|D f^{-1} / E_{x}^{u}\right\|^{\prime} \leq e^{-\mu^{\prime}}$;

(b) $\frac{1}{\sqrt{d}}\|v\|_{x} \leq\|v\|_{x}^{\prime} \leq C e^{\varepsilon k}\|v\|_{x}, \forall v \in T_{x} M, x \in \Lambda_{k}$, where $C=\frac{2}{1-e^{-\varepsilon}}$.

In $\S 2.1$ through $\S 2.5$, the diffeomorphism $f$ is supposed to be $C^{1}$. From now on, we assume that $f$ is $C^{1+\alpha}, 0<\alpha<1$; that is, $f$ is $C^{1}$ and furthermore there exists a constant $K>0$ so that

$$
\left\|D_{x} f-D_{y} f\right\| \leq K d(x, y)^{\alpha}, \quad \forall x, y \in M,
$$

provided $d(x, y)$ is small.

2.6. Extension of Lyapunov metric ([9]-[11]). Fix a point $x \in \Lambda=\Lambda(\lambda, \mu, \epsilon)$, where $\Lambda(\lambda, \mu, \epsilon)$ is a Pesin set; see $\S 2.4$. By taking charts about $x, f(x) \in M$ we can assume without loss of generality that $x \in \mathbb{R}^{d}, f(x) \in \mathbb{R}^{d}$. For a sufficiently small neighborhood $U$ of $x$, we can trivialize the tangent bundle over $U$ by identifying $T_{U} M \equiv U \times \mathbb{R}^{d}$. For any point $y \in U$ and tangent vector $v \in T_{y} M$ we can then use the identification $T_{U} M \equiv U \times \mathbb{R}^{d}$ to translate the vector $v$ to a corresponding vector $\bar{v} \in T_{x} M$. We then define $\|v\|_{y}^{\prime \prime}=\|\bar{v}\|_{x}^{\prime}$, where $\|\cdot\|^{\prime}$ indicates the Lyapunov metric defined in $\S 2.5$. This defines a new norm \|\|$^{\prime \prime}$ on $T_{U} M$ (which agrees with \|\|$^{\prime}$ on the fiber $T_{x} M$ ). Similarly, we can define \|\|$_{z}^{\prime \prime}$ on $T_{z} M$ (for any $z$ in a 
sufficiently small neighborhood of $f(x)$ ). We write $\bar{v}$ as $v$ whenever there is no confusion. We can define a new splitting $T_{y} M=E_{y}^{s^{\prime}} \oplus E_{y}^{u^{\prime}}, y \in U$ by translating the splitting $T_{x} M=E_{x}^{s} \oplus E_{x}^{u}$ (and similarly for $T_{z} M=E_{z}^{s^{\prime}} \oplus E_{z}^{u^{\prime}}$ ).

There exist $0<\lambda^{\prime \prime}<\lambda^{\prime}, 0<\mu^{\prime \prime}<\mu^{\prime}$ and $\varepsilon_{0}>0$ such that if we set $\varepsilon_{k}=\varepsilon_{0} e^{-\varepsilon k}$, then for any $y \in B\left(x, \varepsilon_{k}\right)$ in an $\varepsilon_{k}$ neighborhood of $x \in \Lambda_{k}$ we have a splitting $T_{y} M=E_{y}^{s^{\prime}} \oplus E_{y}^{u^{\prime}}$ with hyperbolicity behavior:

$\left\|D_{y} f(v)\right\|_{f y}^{\prime \prime} \leq e^{-\lambda^{\prime \prime}}\|v\|_{y}^{\prime \prime}$, for every $v \in E_{y}^{s^{\prime}}$ $\left\|D_{y} f^{-1}(w)\right\|_{f^{-1} y}^{\prime \prime} \leq e^{-\mu^{\prime \prime}}\|w\|_{y}^{\prime \prime}$, for every $w \in E_{y}^{u^{\prime}}$.

The constant $\epsilon_{0}$ here and afterwards depends on various global properties of $f$, e.g., the Hölder constants, the size of the local trivialization; see p. 73 in [12].

2.7. Shadowing lemma and closing lemma. Let $\left(\delta_{k}\right)_{k=1}^{+\infty}$ be a sequence of positive real numbers. Let $\left(x_{n}\right)_{n=-\infty}^{+\infty}$ be a sequence in $\Lambda=\Lambda(\lambda, \mu, \epsilon)$ for which there exists a sequence $\left(s_{n}\right)_{n=-\infty}^{+\infty}$ of positive integers satisfying:

(a) $x_{n} \in \Lambda_{s_{n}}, \forall n \in \mathbb{Z}$

(b) $\left|s_{n}-s_{n-1}\right| \leq 1, \forall n \in \mathbb{Z}$;

(c) $d\left(f x_{n}, x_{n+1}\right) \leq \delta_{s_{n}}, \forall n \in \mathbb{Z}$

then we call $\left(x_{n}\right)_{n=-\infty}^{+\infty}$ a $\left(\delta_{k}\right)_{k=1}^{+\infty}$ pseudo-orbit. Given $\eta>0$, a point $x \in M$ is an $\eta$-shadowing point for the $\left(\delta_{k}\right)_{k=1}^{+\infty}$ pseudo-orbit if $d\left(f^{n} x, x_{n}\right) \leq \eta \varepsilon_{s_{n}}, \forall n \in \mathbb{Z}$, where $\varepsilon_{k}=\varepsilon_{0} e^{-\varepsilon k}$.

Lemma 2.1 (Shadowing lemma [5], [12, Thm. 5.1]). Let $f: M \rightarrow M$ be a $C^{1+\alpha}$ diffeomorphism, with a nonempty Pesin set $\Lambda=\Lambda(\lambda, \mu ; \varepsilon)$ and fixed parameters, $\lambda, \mu \gg \varepsilon>0$. For $\forall \eta>0$ there exists a sequence $\left(\delta_{k}\right)_{k=1}^{+\infty}$ such that for any $\left(\delta_{k}\right)_{k=1}^{+\infty}$ pseudo-orbit there exists a unique $\eta$-shadowing point.

Remark. If we change $\varepsilon_{0} e^{-a k}$ for $\varepsilon_{k}=\epsilon_{0} e^{-\epsilon k}$, where $\min (\lambda-2 \epsilon, \mu-2 \varepsilon)>a \geq \varepsilon$, then the shadowing lemma is still true (see the argument on pages 89-93 in [12]).

Lemma 2.2 (Closing lemma [5]). Let $f: M \rightarrow M$ be a $C^{1+\alpha}$ diffeomorphism and let $\Lambda=\Lambda(\lambda, \mu ; \varepsilon)$ be a nonempty Pesin set. For $\forall k \geq 1,0<\eta<1$, there exists $\beta=\beta(k, \eta)>0$ such that if $x, f^{p} x \in \Lambda_{k}$ and $d\left(x, f^{p} x\right)<\beta$, then there exists a periodic point $z \in M$, with $z=f^{p} z$ and $d(z, x)<\eta$.

Remark. By the shadowing lemma (Lemma 2.1) and its remark, we easily get a more convenient version of the closing lemma as follows:

Let $f: M \rightarrow M$ be a $C^{1+\alpha}$ diffeomorphism and let $\Lambda=\Lambda(\lambda, \mu ; \varepsilon)$ be a nonempty Pesin set. For $\forall k \geq 1,0<\eta<1, \min (\lambda-2 \varepsilon, \mu-2 \varepsilon)>\theta \geq \varepsilon$, there exists $\beta=\beta(k, \eta, \theta)>0$ with the property that if $x, f^{p} x \in \Lambda_{k}$ and $d\left(x, f^{p} x\right)<\beta$, then there exists a periodic point $z \in M, z=f^{p} z$, such that $d\left(f^{i} x, f^{i} z\right)<\eta \varepsilon_{0} e^{-\theta i}$, for $0 \leq i \leq p-1$ (see p. 95 in [12]).

\section{The Largest and the Smallest Lyapunov exponents}

In this section we show that the largest and the smallest Lyapunov exponents of an ergodic hyperbolic measure are approximated by those of hyperbolic periodic orbits.

Theorem 3.1. Let $f: M \rightarrow M$ be a $C^{1+\alpha}, 0<\alpha<1$, diffeomorphism of a compact manifold of dimension $d$, and let $m$ be an ergodic hyperbolic measure with Lyapunov exponents $\lambda_{1}<\cdots<\lambda_{r}<0<\lambda_{r+1}<\cdots<\lambda_{t}(t \leq d)$. Then the largest 
Lyapunov exponent $\lambda_{t}$ can be approximated by the largest Lyapunov exponents of hyperbolic periodic orbits. More precisely, for any $\gamma>0$, there exists a hyperbolic periodic point $z$ with Lyapunov exponents $\lambda_{1}^{z} \leq \ldots \leq \lambda_{d}^{z}$ such that $\left|\lambda_{t}-\lambda_{d}^{z}\right|<\gamma$.

Before proving Theorem 3.1, we explain the main idea. By using Katok's shadowing lemma we get a hyperbolic periodic orbit to trace a certain segment of orbit in a Pesin set of $m$. The orbit segment is uniformly hyperbolic under the Lyapunov metric, and the norm of $D f$ (which relates closely to the largest exponent) when restricted on the orbit segment can be controlled very well by the Lyapunov exponent under a suitable Lyapunov metric. Now that the periodic orbit is in a small neighborhood of the orbit segment it traces and $f$ is $C^{1+\alpha}$, we then transfer the counting Lyapunov exponent from the periodic orbit to the orbit segment. This enables us to compare the two largest Lyapunov exponents and to estimate their difference.

The proof is somehow technical. We define one Pesin set for $m$ and three Lyapunov metrics for the Pesin set by using all the individual exponents and thus all the corresponding individual subbundles in the Oseledec splitting, comparing with the standard Pesin set as in $\S 2.4$ and the standard Lyapunov metric as in $\S 2.5$ by using the largest negative exponent and the smallest positive exponent and thus the stable bundle that is the direct sum of subbundles corresponding to all negative exponents and the unstable bundle that is the direct sum of the subbundles corresponding to all positive exponents. One of the advantages of our definitions is that they enable us to control the norm of the derivative restricted on each subbundle by the corresponding exponent from both the lower side and the upper side. Another advantage is that we get three pairs of desired estimates (3.1.1)-(3.1.2), (3.2.1)(3.2.2) and (3.3.1)-(3.3.2) under new metrics, comparing with the inequalities (a), (b) under the standard Lyapunov metric as in $\S 2.5$. By using (3.1.1)-(3.1.2) and Katok's closing lemma and criterion in $\S 2.1$ we prove the existence of a periodic orbit $\operatorname{orb}(z)$ which is hyperbolic under the first Lyapunov metric we defined in the proof. (3.2.1)-(3.2.2) contribute to proving that

$$
\lim _{n \rightarrow+\infty} \frac{\log \left\|D_{z} f^{n}\right\|^{(4)}}{n}<\lambda_{t}+\gamma,
$$

where $\|\cdot\|^{(4)}$ denotes the extension metric to the second Lyapunov metric we defined in the proof. (3.2.3)-(3.3.2) contribute to proving that

$$
\lambda_{t}-\gamma<\lim _{n \rightarrow+\infty} \frac{\log \left\|D_{z} f^{n}\right\|^{(6)}}{n},
$$

where $\|\cdot\|^{(6)}$ denotes the extension metric to the third Lyapunov metric we defined in the proof. These inequalities give rise to the final inequality

$$
\left|\lambda_{t}-\lambda_{d}^{z}\right|<\gamma
$$

under the Riemannian metric, although the three metrics we defined are not equivalent to the Riemannian one on a whole Pesin set in general. This is because in our case only a finite number of Pesin blocks are used, and thus the Lyapunov metrics restricted on these blocks are equivalent to the Riemannian one.

The proof is divided into three steps. The existence of a hyperbolic orbit $\operatorname{Orb}(z)$ in step 1 is not a new result; it was proved in Katok 5. Also, our proof is not quite different from in [5]. But it is better adapted to the proof of the following two steps. 
Proof of Theorem 3.1. Given $\min _{1 \leq i \neq j \leq t}\left|\lambda_{i}-\lambda_{j}\right| \gg \varepsilon>0$, and for all $k \in \mathbb{Z}^{+}$, we define $\Lambda_{k}=\Lambda_{k}\left(\left\{\lambda_{1}, \ldots, \lambda_{t}\right\} ; \varepsilon\right)$ to be all points $x \in M$ for which there is a splitting $T_{x} M=E_{x}^{1} \oplus \cdots \oplus E_{x}^{t}$ with

$$
\lim _{n \rightarrow \infty} \frac{\log \left\|D f^{n} v\right\|}{n}=\lambda_{i}, 0 \neq v \in E_{x}^{i}
$$

and with the invariant property $\left(D_{x} f^{m}\right) E_{x}^{i}=E_{f^{m} x}^{i}, 1 \leq i \leq t$ and satisfying:

(a) $e^{-\varepsilon k} e^{\left(\lambda_{i}-\varepsilon\right) n} e^{-\varepsilon|m|} \leq\left\|D f^{n} / E_{f}^{i} m_{x}\right\| \leq e^{\varepsilon k} e^{\left(\lambda_{i}+\varepsilon\right) n} e^{\varepsilon|m|}, 1 \leq i \leq t, \forall m \in$ $\mathbb{Z}, n \geq 1$

(b) $\tan \left(\operatorname{Angle}\left(E_{f^{m} x}^{i}, E_{f^{m} x}^{j}\right)\right) \geq e^{-\varepsilon k} e^{-\varepsilon|m|}, \forall i \neq j, \forall m \in \mathbb{Z}$.

We set $\Lambda=\Lambda\left(\left\{\lambda_{1}, \ldots, \lambda_{t}\right\} ; \varepsilon\right)=\bigcup_{k=1}^{+\infty} \Lambda_{k}$ and call $\Lambda$ a Pesin set. We easily get that $m(\Lambda)=1$. This Pesin set is slightly different from the standard one as in $\S 2.4$, but the properties(1)-(4) stated there are still true.

Let $q=\min _{1 \leq i \neq j \leq t}\left|\lambda_{i}-\lambda_{j}\right|$, and take arbitrarily $\gamma>0$, satisfying $\min \left\{\frac{1}{2}, \frac{5}{d}\right.$, $\left.\frac{q}{2}, \lambda_{t}\right\}>\gamma>0$, and satisfying $\log \frac{\frac{2(t-1) \gamma}{5}+1}{\left(1-\frac{2 \gamma}{5}\right)\left(1-\frac{t \gamma}{5}\right)}<q$. Let

$$
\varepsilon \leq \min \left\{\frac{\gamma}{5}, \frac{1}{4} q-\frac{1}{4} \log \frac{\frac{2(t-1) \gamma}{5}+1}{\left(1-\frac{2 \gamma}{5}\right)\left(1-\frac{t \gamma}{5}\right)}\right\}
$$

and $q \gg \varepsilon>0$.

We divide the proof into three steps.

Step 1. We prove the existence of hyperbolic periodic points near our Pesin set $\Lambda$. Although the way that Katok [5] proved the existence of hyperbolic periodic points near the standard Pesin set as in $\S 2.4$ works in our case here, we present a short proof of the existence of periodic points near our Pesin set by the shadowing lemma and prove the hyperbolicity of these periodic points by the criterion in $\S 2.1$, a slightly different method from that in [5]. The techniques and inequalities developed while proving the hyperbolicity turn out to be helpful to our consecutive steps.

Let $\lambda_{i}^{\prime}=\left|\lambda_{i}\right|-2 \varepsilon$. Then $\lambda_{i}^{\prime}>0$. We define a new norm $\left\|v_{i}\right\|_{i}$ on the spaces $E_{x}^{i}$, $1 \leq i \leq t, x \in \Lambda$. For $v_{i} \in E_{x}^{i}, 1 \leq i \leq r$, we define $\left\|v_{i}\right\|_{i}=\sum_{n=0}^{+\infty} e^{\lambda_{i}^{\prime} n}\left\|D_{x} f^{n}\left(v_{i}\right)\right\|$; for $v_{j} \in E_{x}^{j}, r+1 \leq j \leq t$, we define $\left\|v_{j}\right\|_{j}=\sum_{n=0}^{+\infty} e^{\lambda_{j}^{\prime} n}\left\|D_{x} f^{-n}\left(v_{j}\right)\right\|$. All these series are convergent. For $v=\sum_{i=1}^{t} v_{i}, v_{i} \in E_{x}^{i}$, we define $\|v\|^{(1)}=\max _{1 \leq i \leq t}\left\{\left\|v_{i}\right\|_{i}\right\}$. The norm \|\|$^{(1)}$ is called in the present paper the Lyapunov metric number 1, which coincides with the standard Lyapunov metric in $\S 2.5$ when $\operatorname{dim} M \leq 2$. This is not equivalent to the Riemannian metric in general. With this norm, $f: \Lambda \rightarrow \Lambda$ is uniformly hyperbolic. The following estimates are similar to those in $\S 2.5$.

$$
\begin{gathered}
\left\|D f /_{E_{x}^{i}}\right\|^{(1)} \leq e^{-\lambda_{i}^{\prime}}, 1 \leq i \leq r,\left\|D f^{-1} / E_{x}^{j}\right\|^{(1)} \leq e^{-\lambda_{j}^{\prime}}, r+1 \leq j \leq t ; \\
\frac{1}{\sqrt{d}}\|v\|_{x} \leq\|v\|_{x}^{(1)} \leq C e^{\varepsilon k}\|v\|_{x}, \forall v \in T_{x} M, x \in \Lambda_{k},
\end{gathered}
$$

where $C=\frac{2}{1-e^{-\varepsilon}}$. By $\S 2.6$ one extends this norm to a norm \|\|$^{(2)}$.

From continuity of the Riemannian metric, there exists $\delta>0$ such that

$$
\frac{1}{1+\gamma}<\frac{\|\|_{x}}{\|\|_{y}}<1+\gamma
$$

provided $d(x, y)<\delta$. Fix $\alpha_{0}$ with $\min \left(\lambda_{r+1}-2 \varepsilon,\left|\lambda_{r}\right|-2 \varepsilon\right)>\alpha_{0}>2 \frac{\varepsilon}{\alpha}$, where $\alpha$ is the Hölder constant of $D f$. Since $m\left(\Lambda\left(\left\{\lambda_{1}, \ldots, \lambda_{t}\right\} ; \varepsilon\right)\right)=1$, there exists $k_{0} \in \mathbb{N}$ such that $m\left(\Lambda_{k_{0}}\right)>0$. For a given arbitrary $\eta>0$, we choose $\beta=\beta\left(k_{0}, \eta, \alpha_{0}\right)>0$ 
as in the remark to Lemma 2.2 with $\beta<\delta$. There exists $y_{0} \in \Lambda_{k_{0}}$ such that $m\left(B\left(y_{0}, \frac{\beta}{2}\right) \cap \Lambda_{k_{0}}\right)>0$ by compactness of $\Lambda_{k_{0}}$. By Poincaré's recurrence theorem, $\exists y \in B\left(y_{0}, \frac{\beta}{2}\right) \cap \Lambda_{k_{0}}$, and $\exists p>1$ such that $f^{p} y \in B\left(y_{0}, \frac{\beta}{2}\right) \cap \Lambda_{k_{0}}$. Since $d\left(y, f^{p} y\right)<$ $\beta$ and $\min \left(\lambda_{r+1}-2 \epsilon,\left|\lambda_{r}\right|-2 \epsilon\right)>\alpha_{0}>2 \frac{\varepsilon}{\alpha}>\varepsilon$, by Lemma 2.2 and its remark there exists a periodic point $z \in M, z=f^{p} z$, with

$$
d\left(f^{i} y, f^{i} z\right)<\eta \varepsilon_{0} e^{-\alpha_{0} i}, \quad 0 \leq i \leq p-1 .
$$

For $\forall v \in T_{f^{i} y} M, 0 \leq i \leq p-2$, by definition of \|\|$^{(2)}$ we have

$$
\left\|D_{f^{i} z} f v-D_{f^{i} y} f v\right\|_{f^{i+1} y}^{(2)}=\left\|D_{f^{i} z} f v-D_{f^{i} y} f v\right\|_{f^{i+1} y}^{(1)} .
$$

Using (3.1.2), (3.1.4) and noting $f^{i+1} y \in \Lambda_{k_{0}+i+1}$, we have

$$
\begin{gathered}
\left\|D_{f^{i} z} f v-D_{f^{i} y} f v\right\|_{f^{i+1} y}^{(1)} \\
\leq \quad C e^{\varepsilon\left(k_{0}+i+1\right)}\left\|D_{f^{i} z} f v-D_{f^{i} y} f v\right\|_{f^{i+1} y} \\
\leq \quad \\
\leq \quad C e^{\varepsilon\left(k_{0}+i+1\right)} K d\left(f^{i} z, f^{i} y\right)^{\alpha}\|v\|_{f^{i} y} \\
\leq \quad C \sqrt{d} e^{\varepsilon\left(k_{0}+1\right)} K \eta^{\alpha} \varepsilon_{0}^{\alpha} e^{-\left(\alpha_{0} \alpha-\varepsilon\right) i}\|v\|_{f^{i} y}^{(2)},
\end{gathered}
$$

and thus

$$
\left\|D_{f^{i} z} f-D_{f^{i} y} f\right\|^{(2)} \leq C \sqrt{d} e^{\varepsilon\left(k_{0}+1\right)} K \eta^{\alpha} \varepsilon_{0}^{\alpha} e^{-\left(\alpha_{0} \alpha-\varepsilon\right) i}, 0 \leq i \leq p-2 .
$$

Similarly, for $f^{-1}$ and $v \in T_{f^{i} y} M, 1 \leq i \leq p-1$, we have by (3.1.2) and (3.1.4),

$$
\begin{array}{cc} 
& \left\|D_{f^{i} z} f^{-1} v-D_{f^{i} y} f^{-1} v\right\|_{f^{i-1} y}^{(2)} \\
\leq & C e^{\varepsilon\left(k_{0}+i-1\right)}\left\|\left(D_{f^{i-1} z} f\right)^{-1}\left(D_{f^{i-1} y} f-D_{f^{i-1} z} f\right)\left(D_{f^{i-1} y} f\right)^{-1}(v)\right\|_{f^{i-1} y} \\
\leq & C e^{\varepsilon\left(k_{0}+i-1\right)} K \sqrt{d}\left\|\left(D_{f^{i-1} z} f\right)^{-1}\right\|\left\|\left(D_{f^{i-1} y} f\right)^{-1}\right\| d\left(f^{i-1} z, f^{i-1} y\right)^{\alpha}\|v\|_{f^{i} y}^{(1)} \\
\leq & C e^{\varepsilon k_{0}} K \sqrt{d} \eta^{\alpha}\left\|D f^{-1}\right\|^{2} \varepsilon_{0}^{\alpha} e^{-\left(\alpha_{0} \alpha-\varepsilon\right)(i-1)}\|v\|_{f^{i} y}^{(2)},
\end{array}
$$

and thus

(3.1.6)

$\left\|D_{f^{i} z} f^{-1}-D_{f^{i} y} f^{-1}\right\|^{(2)} \leq C e^{\varepsilon k_{0}} K \sqrt{d} \eta^{\alpha}\left\|D f^{-1}\right\|^{2} \varepsilon_{0}^{\alpha} e^{-\left(\alpha_{0} \alpha-\varepsilon\right)(i-1)}, 1 \leq i \leq p-1$.

For $v \in T_{f^{p-1} y} M$, by (3.1.3) we have

$$
\begin{array}{cc} 
& \left\|D_{f^{p-1} z} f v-D_{f^{p-1} y} f v\right\|_{f^{p} y}^{(2)} \\
\leq & C e^{\varepsilon k_{0}}\left\|D_{f^{p-1} z} f v-D_{f^{p-1} y} f v\right\|_{y} \\
\leq & (1+\gamma) C e^{\varepsilon k_{0}}\left\|D_{f^{p-1} z} f v-D_{f^{p-1} y} f v\right\|_{f^{p} y} \\
\leq & (1+\gamma) K C e^{\varepsilon k_{0}} d\left(f^{p-1} z, f^{p-1} y\right)^{\alpha}\|v\|_{f^{p-1} y} \\
\leq & (1+\gamma) \sqrt{d} K C \eta^{\alpha} \varepsilon_{0}^{\alpha} e^{\varepsilon k_{0}} e^{-\alpha_{0} \alpha(p-1)}\|v\|_{f^{p-1} y}^{(2)},
\end{array}
$$

and thus

$$
\left\|D_{f^{p-1} z} f-D_{f^{p-1} y} f\right\|^{(2)} \leq(1+\gamma) \sqrt{d} K C \eta^{\alpha} \varepsilon_{0}^{\alpha} e^{\varepsilon k_{0}} e^{-\alpha_{0} \alpha(p-1)} .
$$

For $v \in T_{f^{p} y} M$, we have by using similar estimates as above,

$$
\left\|D_{z} f^{-1}-D_{f^{p} y} f^{-1}\right\|^{(2)} \leq(1+\gamma) C \eta^{\alpha} \varepsilon_{0}^{\alpha} e^{\varepsilon k_{0}} K \sqrt{d}\left\|D f^{-1}\right\|^{2} e^{-\left(\alpha_{0} \alpha-\varepsilon\right)(p-1)} .
$$


Let

$$
\begin{aligned}
& B=\max \left\{C e^{\varepsilon\left(k_{0}\right)} K \sqrt{d}\left\|D f^{-1}\right\|^{2} \varepsilon_{0}^{\alpha},(1+\gamma) C \varepsilon_{0}^{\alpha} e^{\varepsilon k_{0}} K \sqrt{d}\left\|D f^{-1}\right\|^{2},\right. \\
& \left.C \sqrt{d} e^{\varepsilon\left(k_{0}+1\right)} K \varepsilon_{0}^{\alpha},(1+\gamma) \sqrt{d} K C \varepsilon_{0}^{\alpha} e^{\varepsilon k_{0}}\right\} .
\end{aligned}
$$

From (3.1.5)-(3.1.9) we have

$$
\left\|D_{f^{i} z} f-D_{f^{i} y} f\right\|^{(2)} \leq B \eta^{\alpha} e^{-\left(\alpha_{0} \alpha-\varepsilon\right) i}, 0 \leq i \leq p-1,
$$

and

$$
\left\|D_{f^{i} z} f^{-1}-D_{f^{i} y} f^{-1}\right\|^{(2)} \leq B \eta^{\alpha} e^{-\left(\alpha_{0} \alpha-\varepsilon\right)(i-1)}, 1 \leq i \leq p,
$$

where we remember from the choice of $\alpha_{0}$ that $\alpha_{0} \alpha-\varepsilon>0$.

Let

$$
E_{x}^{s}=E_{x}^{1} \oplus \cdots \oplus E_{x}^{r}, \quad E_{x}^{u}=E_{x}^{r+1} \oplus \cdots \oplus E_{x}^{t} .
$$

Let $A=\max \left(e^{\lambda_{r}+2 \varepsilon}, e^{-\lambda_{r+1}+2 \varepsilon}\right)$. Consider a system of inequalities:

or

$$
\left\{\begin{array}{c}
x<1-A-x \\
x<\frac{1}{A+x}-1
\end{array}\right.
$$

$$
\left\{\begin{array}{c}
x<\frac{1-A}{2} \\
x^{2}+(1+A) x+A-1<0 .
\end{array}\right.
$$

Since $A<1$, there exists a real number $b>0$ such that any number included in $(0, b)$ is a solution of the system. Now we make a restriction that $\eta \leq\left(\frac{b}{B}\right)^{\frac{1}{\alpha}}$ (we will make another restriction in Step 2). Then $B \eta^{\alpha}$ is a solution of the system, i.e.

$$
\left\{\begin{array}{c}
B \eta^{\alpha}<1-\left(A+B \eta^{\alpha}\right) \\
B \eta^{\alpha}<\frac{1}{A+B \eta^{\alpha}}-1
\end{array}\right.
$$

Under the invariant splitting $E_{f^{i} y}^{s} \oplus E_{f^{i} y}^{u} i \in \mathbb{Z}, D_{f^{i} y} f$ and $D_{f^{i} y} f^{-1}$ are diagonal block matrices, and $D_{f^{i} z} f$ and $D_{f^{i} z} f^{-1}$ are block matrices as follows:

$$
\begin{gathered}
D_{f^{i} z} f=\left[\begin{array}{ll}
G_{11}^{i} & G_{12}^{i} \\
G_{21}^{i} & G_{22}^{i}
\end{array}\right]: E_{f^{i} y}^{s} \oplus E_{f^{i} y}^{u} \rightarrow E_{f^{i+1} y}^{s} \oplus E_{f^{i+1} y}^{u}, \\
D_{f^{i} z} f^{-1}=\left[\begin{array}{ll}
G_{11}^{i^{\prime}} & G_{12}^{i^{\prime}} \\
G_{21}^{i^{\prime}} & G_{22}^{i^{\prime}}
\end{array}\right]: E_{f^{i+1} y}^{s} \oplus E_{f^{i+1} y}^{u} \rightarrow E_{f^{i} y}^{s} \oplus E_{f^{i} y}^{u} .
\end{gathered}
$$

By (3.1.1), (3.1.10)-(3.1.12), we have

$$
\begin{gathered}
\max \left(\left\|G_{11}^{i}\right\|^{(2)},\left\|G_{22}^{i^{-1}}\right\|^{(2)},\left\|G_{11}^{i^{-1}}\right\|^{(2)},\left\|G_{22}^{i^{\prime}}\right\|^{(2)}\right)<A+B \eta^{\alpha}, \\
\max \left(\left\|G_{21}^{i}\right\|^{(2)},\left\|G_{21}^{i}\right\|^{(2)},\left\|G_{12}^{i^{\prime}}\right\|^{(2)},\left\|G_{21}^{i^{\prime}}\right\|^{(2)}\right)<B \eta^{\alpha} .
\end{gathered}
$$

According to (3.1.13) and the criterion in $\S 2.1$, orb $(z, f)$ is uniformly hyperbolic with the norm \|\|$^{(2)}$. Observe that $\operatorname{or} b(z, f)$ consists of finitely many points, and $\operatorname{orb}(z, f)$ is hyperbolic as well with the Riemannian norm \|\| .

Step 2. We prove that $\lim _{n \rightarrow+\infty} \frac{\log \left\|D_{z} f^{n}\right\|}{n}<\lambda_{t}+\gamma$.

Let $T_{i}=\left(D_{f^{i} y} f\right)^{-1} \circ D_{f^{i} z} f, 0 \leq i \leq p-1$. Then

$$
D_{z} f^{p}=D_{f^{p-1} y} f \circ T_{p-1} \circ \ldots \circ D_{f y} f \circ T_{1} \circ D_{y} f \circ T_{0} .
$$

For $v_{i} \in E_{x}^{i}, 1 \leq i \leq t, x \in \Lambda$, we define $\left\|v_{i}\right\|_{i}^{\prime}=\sum_{n=0}^{+\infty} e^{-\left(\lambda_{i}+2 \varepsilon\right) n}\left\|D_{x} f^{n}\left(v_{i}\right)\right\|$, which are clearly convergent. For $v=\sum_{i=1}^{t} v_{i}, v_{i} \in E_{x}^{i}$, we define $\|v\|^{(3)}=$ 
$\max _{1 \leq i \leq t}\left\{\left\|v_{i}\right\|_{i}^{\prime}\right\}$. We give this norm the Lyapunov metric number 3. This metric coincides with the Lyapunov metric number 1 when restricted in the stable bundle, the direct sum of the subbundles corresponding to the negative exponents. This metric is not equivalent to the Riemannain metric in general. The following estimates are similar to $\S 2.5$ :

$$
\begin{gathered}
\left\|D f / E_{x}^{i}\right\|^{(3)} \leq e^{\lambda_{i}+2 \varepsilon}, 1 \leq i \leq t ; \\
\frac{1}{\sqrt{d}}\|v\|_{x} \leq\|v\|_{x}^{(3)} \leq C e^{\varepsilon k}\|v\|_{x}, \forall v \in T_{x} M, x \in \Lambda_{k},
\end{gathered}
$$

where $C=\frac{2}{1-e^{-\varepsilon}}$. One can extend this norm to a new norm \|\|$^{(4)}$ by $\S 2.6$.

Repeating the process from (3.1.5) to (3.1.11) in Step 1, we obtain

$$
\begin{aligned}
& \left\|D_{f^{i} z} f-D_{f^{i} y} f\right\|^{(4)} \leq B \eta^{\alpha} e^{-\left(\alpha_{0} \alpha-\varepsilon\right) i}, 0 \leq i \leq p-1, \\
& \left\|D_{f^{i} z} f^{-1}-D_{f^{i} y} f^{-1}\right\|^{(4)} \leq B \eta^{\alpha} e^{-\left(\alpha_{0} \alpha-\varepsilon\right)(i-1)}, 1 \leq i \leq p,
\end{aligned}
$$

where $B$ is the same constant as in (3.1.9). From (3.2.2) and (3.2.3), for $0 \leq i \leq p-1$ we have

$$
\begin{aligned}
\left\|T_{i}-I\right\|^{(4)} & =\left\|\left(D_{f^{p-i-1} y} f\right)^{-1} \circ D_{f^{p-i-1} z} f-\left(D_{f^{p-i-1} y} f\right)^{-1} \circ D_{f^{p-i-1} y} f\right\|^{(4)} \\
& \leq B \eta^{\alpha} e^{-\left(\alpha_{0} \alpha-\varepsilon\right)(p-i-1)}\left\|\left(D_{f^{p-i-1} y} f\right)^{-1}\right\|^{(4)} \\
& \leq C B \eta^{\alpha} e^{-\left(\alpha_{0} \alpha-2 \varepsilon\right)(p-i-1)} e^{\left(k_{0}+2\right) \varepsilon}\left\|D_{f^{p-i} y} f^{-1}\right\| .
\end{aligned}
$$

By using an elementary fact that $\log (1+x)<x, \forall x>0$, we have

$$
\left|\log \left\|T_{i}\right\|^{(4)}\right|<\left\|T_{i}-I\right\|^{(4)}<C B \eta^{\alpha} e^{-\left(\alpha_{0} \alpha-2 \varepsilon\right)(p-i-1)} e^{\left(k_{0}+2\right) \varepsilon}\left\|D_{f^{p-i} y} f^{-1}\right\| .
$$

Let $\eta$ be small enough such that

$$
C B \eta^{\alpha} e^{-\left(\alpha_{0} \alpha-2 \varepsilon\right)(p-i-1)} e^{\left(k_{0}+2\right) \varepsilon}\left\|D_{f^{p-i} y} f^{-1}\right\|<\frac{\gamma}{5}, 0 \leq i \leq p-1 .
$$

Observe that for given arbitrary $i \in \mathbb{N}$ there exists $1 \leq j \leq t$ such that $\left\|D_{f^{i} y} f\right\|^{(3)}=\left\|D_{f^{i} y} f /_{E_{f^{i} y}^{j}}\right\|^{\prime}$. From (3.2.1) and (3.2.6) and the choice of $\eta$ we have

$$
\begin{aligned}
& \frac{\log \left\|D_{z} f^{n p}\right\|^{(4)}}{p n}=\frac{\log \left\|\left(D_{z} f^{p}\right)^{n}\right\|^{(4)}}{p n} \leq \frac{\log \left\|D_{z} f^{p}\right\|^{(4)}}{p} \\
\leq & \frac{\log \left(\prod_{i=0}^{p-1}\left\|D_{f^{i} y} f\right\|^{(3)} \prod_{i=0}^{p-1}\left\|T_{i}\right\|^{(4)}\right)}{p} \\
= & \frac{1}{p} \log \left(\prod_{i=0}^{p-1}\left\|D_{f^{i} y} f\right\|^{(3)}\right)+\frac{1}{p} \sum_{i=0}^{p-1} \log \left\|T_{i}\right\|^{(4)} \\
< & \lambda_{t}+2 \varepsilon+\frac{\gamma}{5} .
\end{aligned}
$$

Then we have from the choice of $\varepsilon$,

$$
\lim _{n \rightarrow+\infty} \frac{\log \left\|D_{z} f^{n}\right\|^{(4)}}{n}<\lambda_{t}+\gamma .
$$

Noting that the norm \|\|$^{(4)}$ and the Riemannian norm \|\| are equivalent when restricted on $\operatorname{Orb}(z)$, we get

$$
\lim _{n \rightarrow+\infty} \frac{\log \left\|D_{z} f^{n}\right\|}{n}<\lambda_{t}+\gamma
$$


Step 3. We prove that $\lim _{n \rightarrow+\infty} \frac{\log \left\|D_{z} f^{n}\right\|}{n}>\lambda_{t}-\gamma$.

We now define another norm, by which we emphasize the subbundle corresponding to the largest Lyapunov exponent $\lambda_{t}$. For $v_{i} \in E_{x}^{i}, 1 \leq i \leq t-1, x \in \Lambda$, let

$$
\left\|v_{i}\right\|_{i}^{\prime \prime}=\sum_{n=0}^{+\infty} e^{-\left(\lambda_{i}+2 \varepsilon\right) n}\left\|D_{x} f^{n}\left(v_{i}\right)\right\|
$$

for $v_{t} \in E_{x}^{t}$, let

$$
\left\|v_{t}\right\|_{t}^{\prime \prime}=\sum_{n=0}^{+\infty} e^{\left(\lambda_{t}-2 \varepsilon\right) n}\left\|D_{x} f^{-n}\left(v_{t}\right)\right\| .
$$

All these series are clearly convergent. For $v=\sum_{i=1}^{t} v_{i}, v_{i} \in E_{x}^{i}$, we define $\|v\|^{(5)}=$ $\max _{1 \leq i \leq t}\left\{\left\|v_{i}\right\|_{i}^{\prime \prime}\right\}$. We give this norm the Lyapunov metric number 5. The two Lyapunov metrics, number 3 and number 5 , coincide when restricted to the bundle of the direct sum of subbundles corresponding to all but the largest Lyapunov exponents. Lyapunov metric number 5 is not equivalent to the Riemannian metric in general. The following estimates are clear:

$$
\begin{aligned}
& \left\|D f / E_{x}^{i}\right\|^{(5)} \leq e^{\lambda_{i}+2 \varepsilon}, 1 \leq i \leq t-1,\left\|D f / E_{x}^{t}\right\|^{(5)} \geq e^{\lambda_{t}-2 \varepsilon} \\
& \frac{1}{\sqrt{d}}\|v\|_{x} \leq\|v\|_{x}^{(5)} \leq C e^{\varepsilon k}\|v\|_{x}, \forall v \in T_{x} M, x \in \Lambda_{k},
\end{aligned}
$$

where $C=\frac{2}{1-e^{-\varepsilon}}$. One extends the norm \|\|$^{(5)}$ to a new norm \|\|$^{(6)}$ by $\S 2.6$. Repeating the process from (3.1.5) to (3.1.11) in Step 1, we have

$$
\begin{aligned}
& \left\|D_{f^{i} z} f-D_{f^{i} y} f\right\|^{(6)} \leq B \eta^{\alpha} e^{-\left(\alpha_{0} \alpha-\varepsilon\right) i}, 0 \leq i \leq p-1, \\
& \left\|D_{f^{i} z} f^{-1}-D_{f^{i} y} f^{-1}\right\|^{(6)} \leq B \eta^{\alpha} e^{-\left(\alpha_{0} \alpha-\varepsilon\right)(i-1)}, 1 \leq i \leq p,
\end{aligned}
$$

where $B$ is the same constant as in (3.1.9).

For $\xi>\frac{\frac{2(t-1) \gamma}{5}+1}{1-\frac{2 \gamma}{5}}>1$, let us denote by $K_{\xi}\left(f^{j} y\right)$ the following cones in $T_{f^{j} y} M$, $1 \leq j \leq p-1$ :

$$
K_{\xi}\left(f^{j} y\right)=\left\{\sum_{i=1}^{t} v_{i}, v_{i} \in E_{f^{j} y}^{i}, 1 \leq i \leq t ; \xi\left\|v_{l}\right\|^{(5)}<\left\|v_{t}\right\|^{(5)}, 1 \leq l \leq t-1\right\} .
$$

From (3.3.1) it follows that $D_{f^{j} y} f K_{\xi}\left(f^{j} y\right) \subseteq K_{\xi e^{\lambda_{t}-\lambda_{t-1}-4 \varepsilon}}\left(f^{j+1} y\right)$.

From (3.3.2), (3.3.3) and (3.3.4), repeating the proof from (3.2.5) to (3.2.6) in step 2, we have

$$
\left\|T_{i}-I\right\|^{(6)}<\frac{\gamma}{5}, 0 \leq i \leq p-1 .
$$

Now we consider $T_{j} K_{\xi}\left(f^{j} y\right)$. Let $v \in K_{\xi}\left(f^{j} y\right), \quad v=\sum_{i=1}^{t} v_{i}, \quad v_{i} \in E_{f^{j} y}^{i}$, $\xi\left\|v_{i}\right\|^{(5)} \leq\left\|v_{t}\right\|^{(5)}, \quad 1 \leq i \leq t-1$. From (3.3.5) we have

$$
\frac{\left\|v_{t}\right\|^{(6)}-\sum_{i=1}^{t}\left\|\left(T_{j}-I\right) v_{i}\right\|^{(6)}}{\left\|v_{t}\right\|^{(6)}} \geq \frac{\left\|v_{t}\right\|^{(5)}}{\left\|v_{t}\right\|^{(5)}}-\sum_{i=1}^{t} \frac{\gamma}{5} \frac{\left\|v_{i}\right\|^{(5)}}{\left\|v_{t}\right\|^{(5)}} \geq 1-\frac{t \gamma}{5}
$$


From the choice of $\xi$, for any $1 \leq l \leq t-1$, we have

$$
\begin{aligned}
& \left\|v_{t}\right\|^{(6)}-\sum_{i=1}^{t}\left\|\left(T_{j}-I\right) v_{i}\right\|^{(6)}-\left(\left\|v_{l}\right\|^{(6)}+\sum_{i=1}^{t}\left\|\left(T_{j}-I\right) v_{i}\right\|^{(6)}\right) \\
& \quad \geq\left\|v_{t}\right\|^{(6)}\left(1-\frac{2 \gamma}{5} \sum_{i=1}^{t} \frac{\left\|v_{i}\right\|^{(6)}}{\left\|v_{t}\right\|^{(6)}}-\frac{1}{\xi}\right) \\
& \quad \geq\left\|v_{t}\right\|^{(6)}\left(1-\frac{2 \gamma}{5}-\left(\frac{2(t-1) \gamma}{5}+1\right) \frac{1}{\xi}\right) \\
& \quad>0 .
\end{aligned}
$$

From the definition, the norm \|\|$^{(6)}$ of $T_{j} v$ coincides with that of the projection vector $\left(T_{j} v\right)_{t}$ on $E_{f^{j} y}^{t}$,

$$
\left\|T_{j} v\right\|^{(6)}=\left\|\left(T_{j} v\right)_{t}\right\|_{E_{f^{j} y}^{t}}^{(6)}, \forall v \in K_{\xi}\left(f^{j} y\right), \forall \xi>\frac{\frac{2(t-1) \gamma}{5}+1}{1-\frac{2 \gamma}{5}}>1 .
$$

This implies that

$$
T_{j} K_{\xi}\left(f^{j} z\right) \subseteq K_{1}\left(f^{j} y\right), 0 \leq j \leq p-1 .
$$

Therefore we have

$$
D_{f^{j} y} f T_{j} K_{\xi}\left(f^{j} z\right) \subseteq K_{e^{\lambda_{t}-\lambda_{t-1}-4 \varepsilon}}\left(f^{j+1} y\right), 0 \leq j \leq p-1 .
$$

From the choice of $\gamma$ and $\varepsilon$ we have

$$
e^{\lambda_{t}-\lambda_{t-1}-4 \varepsilon}>\frac{\frac{2(t-1) \gamma}{5}+1}{1-\frac{2 \gamma}{5}}>1
$$

and thus from (3.3.7) we have

$\left\|T_{j+1} v\right\|^{(6)}=\left\|\left(T_{j+1} v\right)_{t}\right\|_{E_{f j+1}^{t}}^{(6)}, \forall j \in \mathbb{N}, v \in K_{e^{\lambda_{t}-\lambda_{t-1}-4 \varepsilon}}\left(f^{j+1} y\right), 0 \leq j \leq p-1$.

From (3.3.6)-(3.3.9) for any $v \in K_{\xi}(y)$ it follows that

$$
\left\|D_{z} f^{j} v\right\|^{(6)}=\left\|\left(D_{z} f^{j} v\right)_{t}\right\|_{E_{f^{j} z}^{t}}^{(6)}, \forall j \in \mathbb{N},
$$

and thus by (3.3.1) and (3.3.6) it follows that

$$
\left\|D_{z} f^{j} v\right\|^{(6)} \geq\left(1-\frac{t \gamma}{5}\right)^{j} e^{\left(\lambda_{t}-2 \varepsilon\right) j}\left\|v_{t}\right\|^{(6)} .
$$

Therefore for $v \in K_{\xi}(y)$ we have by the choice of $\epsilon$,

$$
\lim _{n \rightarrow+\infty} \frac{1}{n} \log \left\|D_{z} f^{n} v\right\|^{(6)} \geq \lambda_{t}-2 \varepsilon+\log \left(1-\frac{t \gamma}{5}\right)>\lambda_{t}-(t+1) \gamma .
$$

Noting that $t \leq d$, we get by replacing $(t+1) \gamma$, by $\gamma$,

$$
\lim _{n \rightarrow+\infty} \frac{1}{n} \log \left\|D_{z} f^{n} v\right\|^{(6)}>\lambda_{t}-\gamma .
$$

Now that the norm \|\|$^{(6)}$ and the Riemannian norm \|\| are equivalent when restricted on $\operatorname{Orb}(z)$, we complete Step 3 by (3.3.10). 
By Step 2 and Step 3 we have

$$
\lambda_{t}-\gamma<\lim _{n \rightarrow+\infty} \frac{\log \left\|D_{z} f^{n}\right\|}{n}<\lambda_{t}+\gamma,
$$

by which we complete Theorem 3.1 .

Theorem 3.2. Let $f: M \rightarrow M$ be a $C^{1+\alpha}$ diffeomorphism of a compact manifold of dimension $d$, and let $m$ be an ergodic hyperbolic measure with Lyapunov exponents $\lambda_{1}<\cdots<\lambda_{r}<0<\lambda_{r+1}<\cdots<\lambda_{t}(t \leq d)$. Then the smallest Lyapunov exponent of $m$ can be approximated by the smallest Lyapunov exponents of hyperbolic periodic orbits. More precisely, for any $\gamma>0$, there exists a hyperbolic periodic point $z$ with Lyapunov exponents $\lambda_{1}^{z} \leq \ldots \leq \lambda_{d}^{z}$ such that $\left|\lambda_{1}-\lambda_{1}^{z}\right|<\gamma$.

Proof. Given $\min _{1 \leq i \neq j \leq t}\left|\lambda_{i}-\lambda_{j}\right| \gg \varepsilon>0$, and for all $k \in \mathbb{Z}^{+}$, we define

$$
\tilde{\Lambda}_{k}=\tilde{\Lambda}_{k}\left(\left\{-\lambda_{1}, \ldots,-\lambda_{t}\right\} ; \varepsilon\right)
$$

to be all points $x \in M$ for which there is a splitting $T_{x} M=E_{x}^{1} \oplus \cdots \oplus E_{x}^{t}$ with

$$
\lim _{n \rightarrow \infty} \frac{\log \left\|d f^{n} v\right\|}{n}=\lambda_{i}, 0 \neq v \in E_{x}^{i}
$$

and with the invariant property $\left(D_{x} f^{m}\right) E_{x}^{i}=E_{f^{m} x}^{i}, 1 \leq i \leq t$ and satisfying:

(a) $e^{-\varepsilon k} e^{\left(-\lambda_{i}-\varepsilon\right) n} e^{-\varepsilon|m|} \leq\left\|D f^{-n} / E_{f}^{i} m_{x}\right\| \leq e^{\varepsilon k} e^{\left(-\lambda_{i}+\varepsilon\right) n} e^{\varepsilon|m|}, 1 \leq i \leq t, \forall m \in$ $\mathbb{Z}, n \geq 1$

(b) $\tan \left(\operatorname{Angle}\left(E_{f^{-m_{x}}}^{i}, E_{f^{-m_{x}}}^{j}\right)\right) \geq e^{-\varepsilon k} e^{-\varepsilon|m|}, \forall i \neq j, \forall m \in \mathbb{Z}$.

We put $\tilde{\Lambda}=\tilde{\Lambda}\left(\left\{-\lambda_{1}, \ldots,-\lambda_{t}\right\} ; \varepsilon\right)=\bigcup_{k=1}^{+\infty} \tilde{\Lambda}_{k}$ and call $\tilde{\Lambda}$ a Pesin set. Clearly $m(\tilde{\Lambda})=1$.

The measure $m$ is ergodic and hyperbolic with respect to $f^{-1}$, for which the Lyapunov exponents are

$$
-\lambda_{1}>\ldots>-\lambda_{r}>0>-\lambda_{r+1}>\ldots>-\lambda_{t} .
$$

By replacing $f$ by $f^{-1}$ in the proof of Theorem 3.1 and by using the Pesin set $\tilde{\Lambda}$ defined above, one can prove Theorem 3.2 , We omit the details.

\section{Proof of Theorem 1.1}

Based on Theorem 3.1 and Theorem 3.2, we prove Theorem 1.1 in this section. We need two more lemmas.

Lemma 4.1. Let $f: M \rightarrow M$ be a $C^{1+\alpha}$ diffeomorphism of a compact manifold of dimension $d$. Let $m$ be an ergodic hyperbolic measure with Lyapunov exponents $\lambda_{1}<\cdots<\lambda_{r}<0<\lambda_{r+1}<\cdots<\lambda_{t}$ together with the associated splitting $E^{1} \oplus \cdots \oplus E^{t}(t \leq d)$. Then the largest Lyapunov exponent of $\left(m, f^{\Lambda^{i}}\right), 1 \leq$ $i \leq \sum_{r+1 \leq j \leq t} \operatorname{dim} E^{j}$ can be approximated by the largest Lyapunov exponent of hyperbolic periodic orbits. More precisely, if we rewrite the Lyapunov spectrum $\left\{\lambda_{1}, \ldots, \lambda_{t}\right\}$ of $(m, f)$ as $\vartheta_{1} \leq \cdots \leq \vartheta_{d}$, then for any $\gamma>0$, there exists a hyperbolic periodic point $z$ with Lyapunov exponents $\lambda_{1}^{z} \leq \ldots \leq \lambda_{d}^{z}$ such that $\mid \sum_{j=d-i+1}^{d} \vartheta_{j}-$ $\sum_{j=d-i+1}^{d} \lambda_{j}^{z} \mid<\gamma$.

Proof. For all $k \in \mathbb{Z}^{+}$, we define

$$
\Lambda_{k}^{i}=\Lambda_{k}^{i}\left(\left\{\sum_{l=1}^{i} \lambda_{j_{l}}, 1 \leq j_{1} \leq j_{2} \leq \ldots \leq j_{i} \leq t\right\} ; \varepsilon\right)
$$


to be all points $x \in M$ for which there is a splitting

$$
\Lambda^{i}(x)=\bigoplus_{1 \leq j_{1} \leq j_{2} \leq \ldots \leq j_{i} \leq t} F_{x}^{j_{1}, \ldots, j_{i}}, \quad F_{x}^{j_{1}, \ldots, j_{i}}=E_{x}^{j_{1}} \wedge \cdots \wedge E_{x}^{j_{i}} \neq 0
$$

with

$$
\lim _{n \rightarrow \infty} \frac{\log \left\|D f^{n^{\Lambda^{i}}}\left(v_{j_{1}} \wedge \ldots \wedge v_{j_{i}}\right)\right\|_{\Lambda^{i}}}{n}=\lambda_{j_{1}}+\ldots+\lambda_{j_{i}}, \quad \forall v_{j_{1}} \wedge \ldots \wedge v_{j_{i}} \in F_{x}^{j_{1}, \ldots, j_{i}}
$$

and with the invariant property $\left(D_{x} f^{m^{\Lambda^{i}}}\right) F_{x}^{j_{1}, \ldots, j_{i}}=F_{f^{m} x}^{j_{1}, \ldots, j_{i}}$ and satisfying

$$
e^{-\varepsilon k} e^{\left(\sum_{l=1}^{i} \lambda_{j_{l}}-\varepsilon\right) n} e^{-\varepsilon|m|} \leq\left\|D f^{n^{\Lambda^{i}}} / F_{f^{j_{1}} \ldots, \ldots, j_{i}}\right\|_{\Lambda^{i}} \leq e^{\varepsilon k} e^{\left(\sum_{l=1}^{i} \lambda_{j_{l}}+\varepsilon\right) n} e^{\varepsilon|m|},
$$

$1 \leq j_{1} \leq j_{2} \leq \ldots \leq j_{i} \leq t, \forall m \in \mathbb{Z}, n \geq 1$.

We put $\Lambda^{i}=\bigcup_{k \geq 1} \Lambda_{k}^{i}$ and call it a Pesin set. Clearly $m\left(\Lambda^{i}\right)=1$. By replacing $f$ by $f^{\Lambda^{i}}$ in the proof of Theorem 3.1 and by using the Pesin set $\Lambda^{i}$ one can prove the Lemma 4.1. We omit the details.

Lemma 4.2. Let $f: M \rightarrow M$ be a $C^{1+\alpha}$ diffeomorphism of a compact manifold of dimension $d$. Let $m$ be an ergodic hyperbolic measure with Lyapunov exponents $\lambda_{1}<\ldots<\lambda_{r}<0<\lambda_{r+1}<\ldots<\lambda_{t}$ together with the associated splitting $E^{1} \oplus \cdots \oplus E^{t}(t \leq d)$. Then the smallest Lyapunov exponents of $\left(m, f^{\Lambda^{i}}\right), 1 \leq$ $i \leq \sum_{1 \leq j \leq r} \operatorname{dim} E^{j}$ can be approximated by the smallest Lyapunov exponent of hyperbolic periodic orbits. More precisely, if we rewrite the Lyapunov spectrum $\left\{\lambda_{1}, \ldots, \lambda_{t}\right\}$ of $(m, f)$ as $\vartheta_{1} \leq \cdots \leq \vartheta_{d}$, then for $\gamma>0$, there exists a hyperbolic periodic point $z$ with Lyapunov exponents $\lambda_{1}^{z} \leq \ldots \leq \lambda_{d}^{z}$ such that $\mid \sum_{j=1}^{i} \vartheta_{j}-$ $\sum_{j=1}^{i} \lambda_{j}^{z} \mid<\gamma$.

Proof. For all $k \in \mathbb{Z}^{+}$, we define

$$
\tilde{\Lambda}_{k}^{i}=\tilde{\Lambda}_{k}^{i}\left(\left\{\sum_{k=1}^{i}\left(-\lambda_{j_{k}}\right), 1 \leq j_{1} \leq j_{2} \leq \ldots \leq j_{i} \leq t\right\} ; \varepsilon\right)
$$

to be all points $x \in M$ for which there is a splitting

$$
\Lambda^{i}(x)=\bigoplus_{1 \leq j_{1} \leq j_{2} \leq \ldots \leq j_{i} \leq t} F_{x}^{\prime j_{1}, \ldots j_{i}}, \quad F_{x}^{\prime j_{1}, \ldots j_{i}}=E_{x}^{j_{1}} \wedge \cdots \wedge E_{x}^{j_{i}} \neq 0
$$

with

$\lim _{n \rightarrow \infty} \frac{\log \left\|d f^{-n^{\Lambda^{i}}}\left(v_{j_{1}} \wedge \ldots \wedge v_{j_{i}}\right)\right\|_{\Lambda^{i}}}{n}=-\left(\lambda_{j_{1}}+\ldots+\lambda_{j_{i}}\right), \quad \forall v_{j_{1}} \wedge \ldots \wedge v_{j_{i}} \in F_{x}^{\prime j_{1}, \ldots, j_{i}}$

and with the invariant property $\left(D_{x} f^{m^{\Lambda^{i}}}\right) F_{x}^{\prime j_{1}, \ldots, j_{i}}=F_{f^{m} x}^{\prime j_{1}, \ldots, j_{i}}$ and satisfying

$$
e^{-\varepsilon k} e^{-\left(\sum_{l=1}^{i} \lambda_{j_{l}}+\varepsilon\right) n} e^{-\varepsilon|m|} \leq\left\|D f^{-n^{\Lambda^{i}}} /{ }_{F_{f}^{\prime} j_{x}}^{\prime j_{1}, \ldots, j_{i}}\right\|_{\Lambda^{i}} \leq e^{\varepsilon k} e^{-\left(\sum_{l=1}^{i} \lambda_{j_{l}}-\varepsilon\right) n} e^{\varepsilon|m|},
$$

$1 \leq j_{1} \leq j_{2} \leq \ldots \leq j_{i} \leq t, \forall m \in \mathbb{Z}, n \geq 1$

We set $\tilde{\Lambda}^{i}=\bigcup_{k \geq 1} \tilde{\Lambda}_{k}^{i}$ and call it a Pesin set. Clearly $m\left(\tilde{\Lambda}^{i}\right)=1$. By replacing $f^{-1}$ by $f^{-\Lambda^{i}}$ in the proof of Theorem 3.2 and by using the Pesin set $\tilde{\Lambda}^{i}$ one can prove Lemma 4.2. We omit the details. 
Proof of Theorem 1.1. We rewrite the Lyapunov spectrum $\left\{\lambda_{1}, \ldots, \lambda_{t}\right\}$ as $\vartheta_{1} \leq$ $\cdots \leq \vartheta_{d}$. We use the notation in the proofs of Theorems 3.13 .2 and Lemmas 4.14 .2 without confusion. For $\forall \gamma>0$, we can choose $\varepsilon$ following the method in Theorem 3.1. Choose $k_{0} \in \mathbb{Z}^{+}$such that

$$
\Gamma_{k_{0}}:=\bigcap_{i=1}^{\sum_{r+1 \leq j \leq t} \operatorname{dim} E^{j}} \Lambda_{k_{0}}^{i} \cap \Lambda_{k_{0}} \cap \tilde{\Lambda}_{k_{0}} \bigcap_{i=1}^{\sum_{1 \leq j \leq r} \operatorname{dim} E^{j}} \tilde{\Lambda}_{k_{0}}^{i}
$$

has positive $m$-measure. Choose $\beta\left(k_{0}, \eta, \alpha_{0}\right)>0$ as in Lemma 2.1 and its remark. According to Theorems 3.13 .2 and Lemmas 4.14 .2 there exists a hyperbolic point $z \in \Gamma_{k_{0}}$ with period $p$ and with Lyapunov exponents $\lambda_{1}^{z} \leq \ldots \leq \lambda_{d}^{z}$ such that

$$
\begin{gathered}
\left|\sum_{j=d-i+1}^{d} \vartheta_{j}-\sum_{j=d-i+1}^{d} \lambda_{d}^{z}\right|<\frac{\gamma}{d}, \quad 1 \leq i \leq \sum_{r+1 \leq j \leq t} \operatorname{dim} E^{i}, \\
\left|\sum_{j=1}^{i} \vartheta_{j}-\sum_{j=1}^{i} \lambda_{j}^{z}\right|<\frac{\gamma}{d}, \quad 1 \leq i \leq \sum_{1 \leq j \leq r} \operatorname{dim} E^{j} .
\end{gathered}
$$

Thus we have

$$
\left|\vartheta_{i}-\lambda_{i}^{z}\right|<\gamma, 1 \leq i \leq d
$$

and this completes the proof.

\section{ACKNOWLEDGEMENT}

The authors thank Shaobo Gan, Chao Liang, Geng Liu and Todd Young very much for their helpful conversations and the referee for his (her) suggestions.

\section{REFERENCES}

[1] L. Barreira, Y. Pesin, Lyapunov exponents and smooth ergodic theory, Univ. Lect. Ser. 23, AMS, Providence, RI, 2002 MR,1862379 (2003a:37040)

[2] L. Barreira, Y. Pesin, Nonuniform hyperbolicity, dynamics of systems with nonzero Lyapunov exponents, Cambridge University Press, Cambridge, 2007 MR 2348606

[3] M. Hirayama, Periodic probability measures are dense in the set of invariant measures, Dist. Cont. Dyn. Sys., 9 (2003), 1185-1192 MR.1974422 (2004a:37032)

[4] M. Hirsch, C. Pugh, Stable manifolds and hyperbolic sets, Proc. Symposia Pure Math. XIV, 133-163, S-S. Chern, S. Smale, Editors, AMS, 1968 MR.0271991 (42:6872)

[5] A. Katok, Lyapunov exponents, entropy and periodic orbits for diffeomorphisms, Publ. Math. IHES, 51 (1980), 137-173 MR.573822 (81i:28022)

[6] A. Katok, L. Mendoza, Dynamical systems with nonuniformly hyperbolic behavior, Supplement to the book: A. Katok, B. Hasselblatt, Introduction to the modern theory of dynamical systems, Cambridge Univ. Press, USA, 1995 MR.1326374 (96c:58055)

[7] C. Liang, G. Liu, W. Sun, Approximation properties on invariant measure and Oseledec splitting in non-uniformly hyperbolic systems, Trans. Amer. Math. Soc. 361 (2009), 15431579. MR2457408

[8] V. I. Oseledec, Multiplicative ergodic theorem, Lyapunov characteristic numbers for dynamical systems, Trans. Moscow Math. Soc., 19 (1968), 197-221 MR0240280 (39:1629)

[9] Y. Pesin, Lyapunov characteristic exponents and ergodic properties of smooth dynamical systems with an invariant measure, Sov. Math. Dok., 17(1976), 196-199

[10] Y. Pesin, Families of invariant manifolds corresponding to nonzero Lyapunov exponents, Izvestija, 10(1976), 1261-1305

[11] Y. Pesin, Characteristic exponents and smooth ergodic theory, Russian Mathematical Surveys, 32 no. 4(1997), 55-114

[12] M. Pollicott, Lectures on ergodic theory and Pesin theory on compact manifolds, Cambridge Univ. Press, 1993 MR 1215938 (94k:58080) 
[13] D. Ruelle, Ergodic theory of differentiable dynamical systems, Pub. Math. Lihes., tome 50 (1979), 27-58 MR.556581 (81f:58031)

[14] K. Sigmund, Generic properties of invariant measures for axiom A diffeomorphisms, Inventiones Math. 11(1970), 99-109 MR0286135(44:3349)

Lmam, School of Mathematical Sciences, Peking University, Beijing 100871, People's Republic of China

E-mail address: wangzq@pku.org.cn

Current address: Department of Mathematics, The Pennsylvania State University, University

Park, Pennsylvania 16802

E-mail address: zuw104@psu.edu

LMam, School of Mathematical Sciences, Peking University, Beijing 100871, People's Republic of China

E-mail address: sunwx@math.pku.edu.cn 